\title{
Effect of temperature and cycle length on microbial competition in PHB-producing sequencing batch reactor
}

\author{
Yang Jiang, Leonie Marang, Robbert Kleerebezem, Gerard Muyzer and \\ Mark C M van Loosdrecht \\ Department of Biotechnology, Delft University of Technology, Julianalaan, The Netherlands
}

\begin{abstract}
The impact of temperature and cycle length on microbial competition between polyhydroxybutyrate (PHB)-producing populations enriched in feast-famine sequencing batch reactors (SBRs) was investigated at temperatures of $20^{\circ} \mathrm{C}$ and $30^{\circ} \mathrm{C}$, and in a cycle length range of $1-18 \mathrm{~h}$. In this study, the microbial community structure of the PHB-producing enrichments was found to be strongly dependent on temperature, but not on cycle length. Zoogloea and Plasticicumulans acidivorans dominated the SBRs operated at $20^{\circ} \mathrm{C}$ and $30{ }^{\circ} \mathrm{C}$, respectively. Both enrichments accumulated PHB more than $75 \%$ of cell dry weight. Short-term temperature change experiments revealed that $P$. acidivorans was more temperature sensitive as compared with Zoogloea. This is particularly true for the PHB degradation, resulting in incomplete PHB degradation in $P$. acidivorans at $20^{\circ} \mathrm{C}$. Incomplete PHB degradation limited biomass growth and allowed Zoogloea to outcompete $P$. acidivorans. The PHB content at the end of the feast phase correlated well with the cycle length at a constant solid retention time (SRT). These results suggest that to establish enrichment with the capacity to store a high fraction of PHB, the number of cycles per SRT should be minimized independent of the temperature.
\end{abstract}

The ISME Journal (2011) 5, 896-907; doi:10.1038/ismej.2010.174; published online 25 November 2010 Subject Category: microbial engineering

Keywords: cycle length; number of cycle per SRT; Plasticicumulans acidivorans; temperature; Zoogloea

\section{Introduction}

Polyhydroxyalkanoates (PHAs) are biodegradable bioplastics that can be produced by bacteria from renewable resources (Kleerebezem and van Loosdrecht, 2007; Keshavarz and Roy, 2010). Since the first discovery of PHA production in Bacillus megaterium by Lemoigne, 1926, over 250 different types of bacteria have been reported as natural PHA producers (Steinbüchel, 1991). In addition to natural producers, genetically-modified organisms are used for the industrial production of PHA (Slater et al., 1988; Braunegg et al, 1998). However, the use of sterile equipment, defined substrates and downstream processing contribute to the high costs of PHA production, which limits the industrial application of PHA (Keshavarz and Roy, 2010).

To reduce the price of bioplastics, the production of PHAs by mixed microbial cultures has been

Correspondence: MCM van Loosdrecht, Department of Biotechnology, Delft University of Technology, Julianalaan 67, Delft, 2628 BC, The Netherlands.

E-mail: M.C.M.vanLoosdrecht@tudelft.nl

Received 26 July 2010; revised 13 September 2010; accepted 13 September 2010; published online 25 November 2010 investigated (Kleerebezem and van Loosdrecht, 2007). This process aims at using non-sterile equipment and wastewater as a substrate, combining wastewater treatment and bioplastic production. A two-step process with a feast-famine regime has been demonstrated to enable the effective enrichment of a PHA-producing mixed microbial culture from the natural environment based on the ecological role of PHA (van Loosdrecht, 2000). However, the maximum PHA content of mixed cultures is in general lower than that of pure cultures (Reis et al., 2003; Dias et al., 2006). This increases the relative costs for downstream processing, and thereby increases the production costs of PHA (Mudliar et al., 2008). Extensive efforts are, therefore being made to improve the PHA content of mixed cultures (Dias et al., 2006). Recently, enrichment of bacteria with a maximum polyhydroxybutyrate (PHB) content of $89 \%$ cell dry weight has been achieved by Johnson et al., 2009a. Molecular, biological and microbiological analyses showed that a novel bacterium Plasticicumulans acidivorans (Jiang et al., submitted) was the dominant PHBproducing bacterium in this enrichment. This $P$. acidivorans mixed culture was initially enriched in a sequencing batch reactor (SBR) operated under 
carbon limitation, at 1 day solid retention time (SRT) and hydraulic retention time, at temperature of $30^{\circ} \mathrm{C}$ and a $12 \mathrm{~h}$ cycle length. The key factors responsible for enrichment of $P$. acidivorans remained unclear. The temperature and the cycle length have been proposed to have a direct impact. However, a detailed evaluation on the effect of temperature and cycle length on the competition of $P$. acidivorans with other bacterial species during the enrichment step was not performed. A thorough understanding of this competition principle would be essential for a commercial success of bioplastic production from waste.

The temperature effects on PHA-producing cultures enriched with a feast-famine strategy have been studied by Krishna and van Loosdrecht, 1999 and by Johnson et al., 2010. However, both of these studies mainly studied the influence of temperature on the kinetics of the process. The effect of temperature on the microbial community structure has been hardly investigated. Regarding the effect of the cycle length of a SBR on PHAproducing mixed cultures, Dionisi et al., 2007 enriched mixed cultures using cycle lengths in a range of 1 to $8 \mathrm{~h}$. Although the microbial composition was analyzed in this study, the competition between the different types of bacterial populations was not explored.

The aim of this study was to analyze the effect of temperature and cycle length on the enrichment of $P$. acidivorans in mixed cultures. SBRs were used to enrich PHB-producing bacteria with a feast-famine regime. Acetate was supplied as the sole carbon source and the polymer produced was consequently pure PHB. Two temperatures $\left(20^{\circ} \mathrm{C}\right.$ and $\left.30^{\circ} \mathrm{C}\right)$ and four different cycle lengths $(1 \mathrm{~h}, 4 \mathrm{~h}, 12 \mathrm{~h}$ and $18 \mathrm{~h}$ ) were tested in this study. Cycle measurements and accumulation experiments were performed when a stable operational performance was established in SBRs. The influence of short-term temperature changes on the dynamics of different enrichments was also studied. Specific conversion rates of all experiments were evaluated with the help of a model developed by Johnson et al., 2009b. Denaturing gradient gel electrophoresis (DGGE) of PCRamplified 16S rRNA gene fragments was used to determine the community composition. Fluorescence in situ hybridization and quantitative PCR were used to determine the relative abundance of the different types of bacteria. These data have been used to discuss the basic mechanism of microbial competition in PHB-accumulating enrichment cultures.

\section{Materials and methods}

Operation of sequencing-batch and fed-batch reactors SBRs were used to enrich the PHB-producing mixed microbial culture. The basic SBR setup was the same as described by Johnson et al., 2009b. The temperature of reactors was set to $20^{\circ} \mathrm{C}$ or $30^{\circ} \mathrm{C}$. Operation of the SBR was based on either $1 \mathrm{~h}, 4 \mathrm{~h}, 12 \mathrm{~h}$ or $18 \mathrm{~h}$ batch cycles consisting of start phase, feeding phase, reaction phase and biomass effluent phase. Totally, six SBRs were carried out at the conditions summarized in Table 1. Two SBRs with $4 \mathrm{~h}$ and $12 \mathrm{~h}$ cycle length were operated at $20^{\circ} \mathrm{C}$ and four SBRs with $1 \mathrm{~h}, 4 \mathrm{~h}, 12 \mathrm{~h}$ and $18 \mathrm{~h}$ cycle lengths were operated at $30^{\circ} \mathrm{C}$. No settling phase was included in the batch cycle. The SRT was therefore equal to the hydraulic retention time, and both were set as 1 day. The $\mathrm{pH}$ of the reactors was maintained at $\mathrm{pH}$ 7. All the reactors were fed with acetate as the sole carbon source and the medium composition was the same as described by Johnson et al., 2009a. The medium for the SBR consisted of a carbon source, a nutrient source and dilution water. The carbon source was $270 \mathrm{~mm} \mathrm{NaAc} \cdot 3 \mathrm{H}_{2} \mathrm{O}$ and the nutrient source was composed of $67.5 \mathrm{mM} \mathrm{NH}_{4} \mathrm{Cl}$, $24.9 \mathrm{mM} \mathrm{KH}_{2} \mathrm{PO}_{4}, 5.5 \mathrm{mM} \mathrm{MgSO}_{4} \cdot 7 \mathrm{H}_{2} \mathrm{O}, 7.2 \mathrm{mM} \mathrm{KCl}$, $15 \mathrm{mll}^{-1}$ trace elements solution according to Vishniac and Santer, 1957 and $100 \mathrm{mg} \mathrm{l}^{-1}$ allythiourea (to prevent nitrification). In each cycle, carbon source, nutrient source and dilution water were dosed into the reactor with a ratio of 1:1:8. The organic loading rate was kept constant at $2.25 \mathrm{Cmmole}^{-1} \mathrm{~h}^{-1}$ for all the reactors.

A fed-batch reactor was used to evaluate the maximum PHB storage capacity of the biomass collected from the SBRs. The experiments were operated at the same temperature as the SRBs from which the biomass was collected. This PHB accumulation experiment was executed under nitrogen-limiting condition. The detailed procedures were the same as described by Johnson et al., 2009b. A total volume of $1 \mathrm{l}$ of acetate- and ammonia- free medium was fed to the reactor filled with $1 \mathrm{l}$ of biomass from previous SBR cycle. The medium composition is $2.49 \mathrm{mM}$ $\mathrm{KH}_{2} \mathrm{PO}_{4}, \quad 0.55 \mathrm{mM} \quad \mathrm{MgSO}_{4} \cdot 7 \mathrm{H}_{2} \mathrm{O}, \quad 0.72 \mathrm{mM} \mathrm{KCl}$, $1.5 \mathrm{mll}^{-1}$ trace elements solution according to Vishniac and Santer, 1957 and $10 \mathrm{mg} \mathrm{l}^{-1}$ allythiourea (to prevent nitification).

\section{Microbial competition experiment}

All conducted SBRs in this study were inoculated by activated sludge from a nutrient removal sewage treatment plant (Kralingseveer, the Netherlands), and operated for 6 days to establish a stable feastfamine regime. To normalize the start point of microbial competition experiment, all SBRs were bioaugmented with $1 \%(\mathrm{w} / \mathrm{w})$ of $P$. acidivoransdominated biomass on the sixth day after the inoculation from activated sludge. In the subsequent 7 days, the biomass from the SBRs was collected for microbial diversity analysis and $P$. acidivorans relative abundance analysis. After this, the SBRs were operated until a stable operational performance was established. The stable operational performance was defined as a stable ammonia concentration and total suspended solids at the end of the cycle and 
898

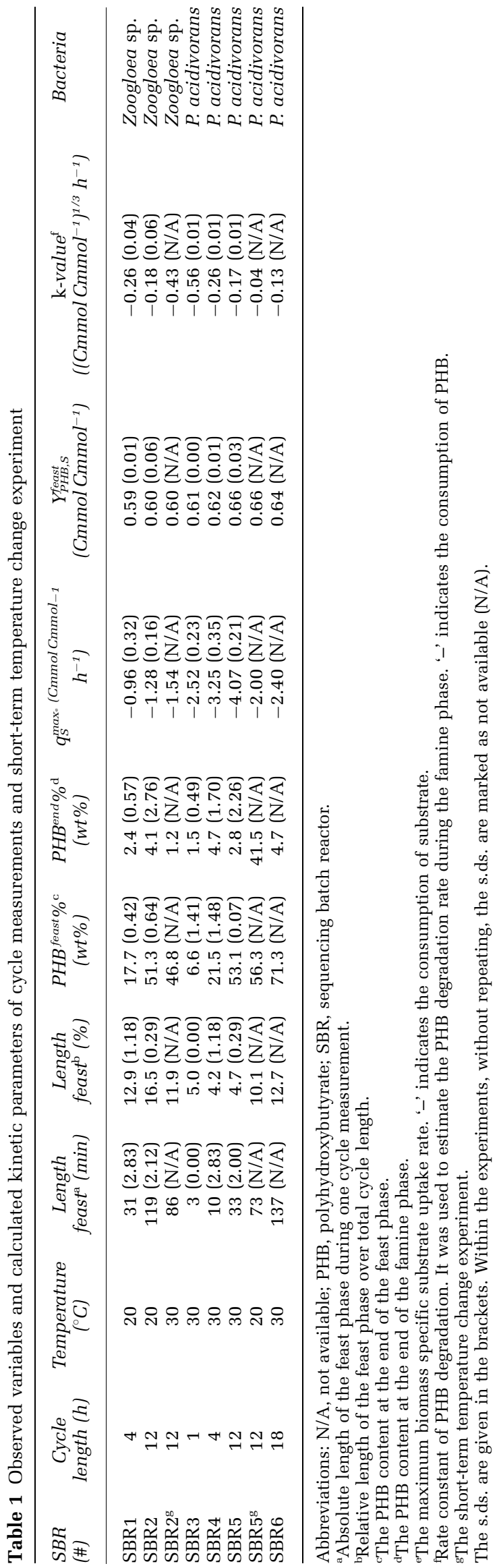

a constant length of the feast phase and $\mathrm{pH}$ profile for at least five SRTs. The biomass from this stable operational stage was collected for cycle measurements, fed-batch experiments or short-term temperature change experiments.

\section{Short-term temperature change experiments}

The effect of a short temperature change on the conversions was evaluated by subjecting the biomass collected from SBR 2 and 5 (Table 1) to a regular cycle but at a different temperature. Effectively the temperature of these two stable SBRs was switched for one cycle to a different temperature and the concentration changes during a cycle were measured.

\section{Data treatment}

During all the cycle measurements and fed-batch experiments, the online data (for example, dissolved oxygen, $\mathrm{pH}$, acid and base dosage, off-gas $\mathrm{CO}_{2}$ and $\mathrm{O}_{2}$ ) were monitored by a personal computer using the software MFCS/win (Sartorius Stedim Systems, Bohemia, NY, USA) and offline data (for example, acetate, total suspended solids, PHB and ammonia) were collected in the same way as described by Johnson et al., 2009b. Samples taken from the reactor for analysis of acetate and ammonia were immediately filtered with a $0.45 \mu \mathrm{m}$ pore size filter (PVDF membrane; Millipore, Tullagreen, County Cork, Ireland). The acetate concentration was determined by highperformance liquid chromatography, using an Aminex HPX-87 H column from Bio-Rad (Hercules, CA, USA) $\left(T=60{ }^{\circ} \mathrm{C}\right)$ coupled to an ultraviolet and a refractive index detector. The total suspended solids were measured by filtration according to standard methods. The PHB concentration was analyzed by gas chromatography (model 6890N, Agilent, Lexington, MA, USA) equipped with a flame ionization detector, on a HP Innowax column. Results of PHB were converted to weight percentage of PHB of the total solids. The model described by Johnson et al., 2009b was used to evaluate the kinetics of the PHB-producing mixed culture on acetate. A constant oxidative phosphorylation efficiency $(\mathrm{P} / \mathrm{O}$ ratio, $\delta$ ) was used to determine the yield coefficients, neglecting a potential small difference caused by the presence of different microorganisms.

\section{Temperature coefficients}

The general activity of the enrichments was demonstrated in terms of the biomass-specific oxygen uptake rate, which was measured by biological oxygen monitor with a temperature range from 15 to $30^{\circ} \mathrm{C}$ and a $\mathrm{pH}$ of 7 . Around $45 \mathrm{ml}$ of biomass suspension at the end of the cycle from SBR 2 and SBR 5 was added into an incubation chamber. Volume of $1 \mathrm{ml}$ of $30 \mathrm{~mm}$ sodium acetate was dosed into the incubation chamber when the temperature was stabilized at the desired value. 
The biomass-specific oxygen consumption rate was calculated from the oxygen depletion rate and biomass concentration in the incubation chamber. Because the biomass suspension was collected at the end of the cycle, the endogenous respiration was assumed negligible.

A simplified Arrhenius equation was used to describe the effect of temperature on the biomassspecific oxygen uptake rate (Equation (1), Brdjanovic et al., 1997). In the equation, $r_{T}$ is the oxygen uptake rate at temperature $T, T$ is the temperature in ${ }^{\circ} \mathrm{C}$ and $\theta$ is the temperature coefficient.

$$
r_{T}=r_{20} \cdot \theta^{(T-20)}
$$

DGGE analysis of PCR-amplified $16 S$ rRNA genes

The microbial composition of all the enrichments was analyzed by PCR-DGGE. Biomass samples were collected from the reactors and were washed with TE buffer to remove culture medium. The genomic DNA was extracted using the Ultra Clean Microbial DNA isolation Kit (MoBio Laboratories, Carlsbad, CA, USA) following the manufacturer's instructions. Subsequently, the extracted DNA was used as template DNA in the PCR. 16S rRNA gene fragments of the different community constituents were obtained by using a ‘touchdown' PCR program with primers $341 \mathrm{~F}$ with a GC clamp and 907R for DGGE analysis (Schäfer and Muyzer, 2001, Table 2).

16S rRNA gene amplicons were applied onto $8 \%$ polyacrylamide gels with denaturing gradient from $20 \%$ to $70 \%$ DNA denaturants $(100 \%$ denaturants is a mixture of $5.6 \mathrm{M}$ urea and $32 \%$ formamide) (Schäfer and Muyzer, 2001). DNA was visualized by ultraviolet illumination after staining with a solution of ethidium bromide and photographed with a digital camera. Individual bands were excised from the gel with a sterile razor blade and incubated overnight in $50 \mu \mathrm{l}$ water at $4{ }^{\circ} \mathrm{C}$.
Re-amplification was performed by using the same primer pair; the purity of excised fragment was checked by DGGE using the same gradient of DNA denaturants. Subsequently, the PCR products were sequenced by a commercial company (Macrogen, Seoul, South Korea). The sequences have been stored in the GenBank under accession numbers: HM627248-HM627253.

\section{Quantification of the bacteria species}

The relative abundance of $P$. acidivorans in all enrichments was roughly estimated by fluorescence in situ hybridization first. The oligonucleotide probes used in this study are listed in Table 2. The general probe mixture EUB338 labeled with Cy5 was used to indicate all bacteria species in the sample. The probe of Beta42a labeled with FLUOS was utilized to describe Betaproteobacteria. To minimize erroneous hybridizations, the unlabeled probe Gamma42a was used to compete with Beta42a probe. A probe specific for $P$. acidivorans (UCB823) labeled with Cy3 was used to determine the abundance of $P$. acidivorans (Johnson et al., 2009a). A probe mixture of ZAL, ZRA and ZBE was used to indicate the abundance of Zoogloea ramigera. The detailed procedure of fluorescence in situ hybridization analysis was described by Johnson et al., 2009a

The relative abundances of $P$. acidivorans in the microbial competition experiment were precisely determined by quantitative PCR. The extracted genomic DNA of each sample was used as DNA template. The primer pair 341F/907R was used for quantifying total bacteria in the SBRs, and the primer pair of UCB127F/UCB823R was used for quantifying $P$. acidivorans (Table 2). Each genomic DNA sample was amplified by both primer pairs to calculate the relative abundance of $P$. acidivorans cells over the total amount of bacteria.

Table 2 Oligonucleotide probes for FISH analysis and primers for PCR-DGGE and qPCR analysis in this study

\begin{tabular}{|c|c|c|c|c|}
\hline Code & Function & Sequence $\left(5^{\prime}-3^{\prime}\right)$ & Specificity & Reference \\
\hline EUB338 & Probe & gctgcctcccgtaggagt & Bacteria & Amann et al. (1990) \\
\hline EUB338II & Probe & gcagccacccgtaggtgt & Bacteria & Daims et al. (1999) \\
\hline EUB338III & Probe & gctgccacccgtaggtgt & Bacteria & Daims et al. (1999) \\
\hline UCB823 & Probe & cctccccaccgtccagtt & P. acidivorans & Johnson et al. (2009a) \\
\hline Beta42a & Probe & gccttcccacttcgttt & Betaproteobacteria & Manz et al. (1992) \\
\hline Gama42a & Probe & gccttcccacatcgttt & Gammaproteobacteria & Manz et al. (1992) \\
\hline ZAL & Probe & cttccatactctaggtac & Zoogloea & Rossello-Mora et al. (1995) \\
\hline ZRA & Probe & ctgccgtactctagttat & Zoogloea & Rossello-Mora et al. (1995) \\
\hline ZBE & Probe & tgccaaactctagccttg & Zoogloea & Rossello-Mora et al. (1995) \\
\hline $341 F-G C$ & Primer & cctacgggaggcagcag ${ }^{\mathrm{a}}$ & Bacteria & Schäfer and Muyzer (2001) \\
\hline $341 \mathrm{~F}$ & Primer & cctacgggaggcagcag & Bacteria & Schäfer and Muyzer (2001) \\
\hline 907R & Primer & ccgtcaattcmtttgagttt & Bacteria & Schäfer and Muyzer (2001) \\
\hline UCB127F & Primer & gaatctgccccacgggtg & P. acidivorans & This study \\
\hline UCB823R & Primer & cctccccaccgtccagtt & P. acidivorans & This study \\
\hline
\end{tabular}

Abbreviations: FISH, fluorescence in situ hybridization; DGGE, denaturing gradient gel electrophoresis; q-PCR, quantitative polymerase chain reaction.

a341F-GC primer contains GC-clamp (cgcccgccgcgccccgcgcccgtcccgecgcccccgcccg) at the $5^{\prime}$-end of the primer. 
900

The quantitative PCR was performed by iQ SYBR Green Supermix Kit (Bio-Rad). Each reaction system was composed of $10 \mu \mathrm{l}$ SYBR Green MasterMix, $0.2 \mu \mathrm{l}$ forward primer, $0.2 \mu \mathrm{l}$ reverse primer, $0.4 \mu \mathrm{l}$ DNA template and $9.2 \mu \mathrm{l} \mathrm{H}_{2} \mathrm{O}$. The following PCR program was used: $5 \mathrm{~min}$ at $95{ }^{\circ} \mathrm{C}$, followed by 40 cycles of $94{ }^{\circ} \mathrm{C}$ for $30 \mathrm{~s}, 57^{\circ} \mathrm{C}$ for $30 \mathrm{~s}$ and $72^{\circ} \mathrm{C}$ for $1 \mathrm{~min}, 72^{\circ} \mathrm{C}$ for $10 \mathrm{~min}$ after the cycle and termination at $4{ }^{\circ} \mathrm{C}$. The DNA of $P$. acidivorans and its 10 , 100 and 1000 times dilutions were used to make the calibration curves from both primer pairs, indicating the relation between the SYBR green fluorescence signal and the quantity of the DNA template. Additionally, the mixtures of $P$. acidivorans DNA and Escherichia coli $5 \alpha(1: 100$ and 1:1000) were used as positive control to check the accuracy and reliability of the quantitative PCR method.

\section{Results}

Performance of the SBRs

A total of six long-term SBRs were conducted to study the impact of temperature and cycle length on enriching PHA-producing microbial cultures. Two temperatures $\left(20^{\circ} \mathrm{C}\right.$ and $\left.30^{\circ} \mathrm{C}\right)$ and four cycle lengths ( $1 \mathrm{~h}, 4 \mathrm{~h}, 12 \mathrm{~h}$ and $18 \mathrm{~h})$ were tested. In all SBRs, a clear feast-famine regime can be observed just one day after the inoculation with activated sludge. Within 2 weeks, a stable operational performance was established in all SBRs. After a stable operational performance was obtained, all SBRs were continuously operated for one more week (seven times of SRT) before performing the cycle measurements and fed-batch experiments. During the cycle, the patterns of substrate consumption and products formation were identical to previously described similar experiments in SBRs (Beun et al., 2002; Johnson et al., 2009a). Acetate was rapidly consumed during the feast phase and used mainly for both growth and PHB production. In the famine phase, the stored PHB was used for biomass synthesis and partly respired to inorganic carbon (Appendix A). The obtained experimental data were evaluated by the metabolic model developed by Johnson et al., 2009b. All important variables measured during the experiments and the parameters estimated from model are listed in Table 1.

As can be seen from Table 1, the length of the feast phase was generally shorter in the SBRs at $30^{\circ} \mathrm{C}$, which was consistent with the higher biomass specific substrate uptake rate in those SBRs. Although the substrate uptake rates differ, the yield of PHB on substrate during the feast phase was always constant (about $0.6 \mathrm{Cmmol} \mathrm{Cmmol}^{-1}$ ). The PHB content at the end of the feast phase was variable, but directly related to the length of the cycle used in the SBR. The PHB content at the end of the famine phase was always low (except for SBR5*), indicating that most of the PHB stored during the feast phase was consumed during the famine phase.
Rate constant of PHB degradation ( $k$-value) characterize the PHB consumption rate during the famine phase. They are listed mainly for the comparison of the short-term temperature change experiments (SBR 2 and SBR $2^{*}$; SBR 5 and SBR $5^{*}$ ). In these four SBRs, higher $k$-values can be observed for SBRs at $30^{\circ} \mathrm{C}$, indicating higher PHB degradation rates.

\section{Microbial composition analysis}

At the same time of performing cycle measurements or fed-batch experiments, a biomass sample was also collected for microbial diversity analysis. DGGE of PCR-amplified 16S rRNA gene fragments was used to determine the community composition of each SBR (Figure 1). Six dominant bands indicated by white dots were excised from the DGGE gel and sequenced. The sequencing results revealed that the SBRs operated at $20^{\circ} \mathrm{C}$ (SBR 1 and SBR 2) were dominated by Zoogloea-related species, whereas $P$. acidivorans dominated the SBRs operated at $30^{\circ} \mathrm{C}$ (SBR 3 to 6). Additionally, some bands labeled by the black dots in SBR 3 to 6 were also analyzed. The sequences of these bands were found identical to $P$. acidivorans. As proposed in Kowalchuk et al., 1997, the generation of multiple banding from a single template could be explained by the use of degenerate primers. During the DGGE analysis in this study, a degenerate nucleotide is present in the reverse primer 907R (Table 2), which could be the reason for the multiple bands representing the same bacterial species on the gel.

Fluorescence in situ hybridization was performed to estimate the relative abundance of the dominant bacteria in the reactors when stable operational performances were established. An oligonucleotide probe specific for $P$. acidivorans (Johnson et al., 2009a) was used to determine the abundance of P. acidivorans. Although specific probes for Zoogloea have been designed (Rossello-Mora et al., 1995), the Zoogloea species enriched in this study showed no hybridization by the specific probes (data not shown). Considering that only one dominant band representing Zoogloea was found in SBR 1 and SBR 2 , the general probe for the Betaproteobacteria was used to estimate the relative abundance of Zoogloea. Consistent with the results of the DGGE, most of the bacteria from the reactors operated at $30^{\circ} \mathrm{C}$ can be hybridized with $P$. acidivorans-specific probe and most of the bacteria from those operated at $20^{\circ} \mathrm{C}$ were hybridized with the probe specific for Betaproteobacteria. This result indicated that temperature had a major impact on microbial composition of the enrichment. In contrast, the cycle length only had a marginal influence on microbial diversity

\section{Microbial competition analysis}

Although the cycle length had very limited impact on the microbial composition when the SBRs stabilized, the influence of the cycle length on the 


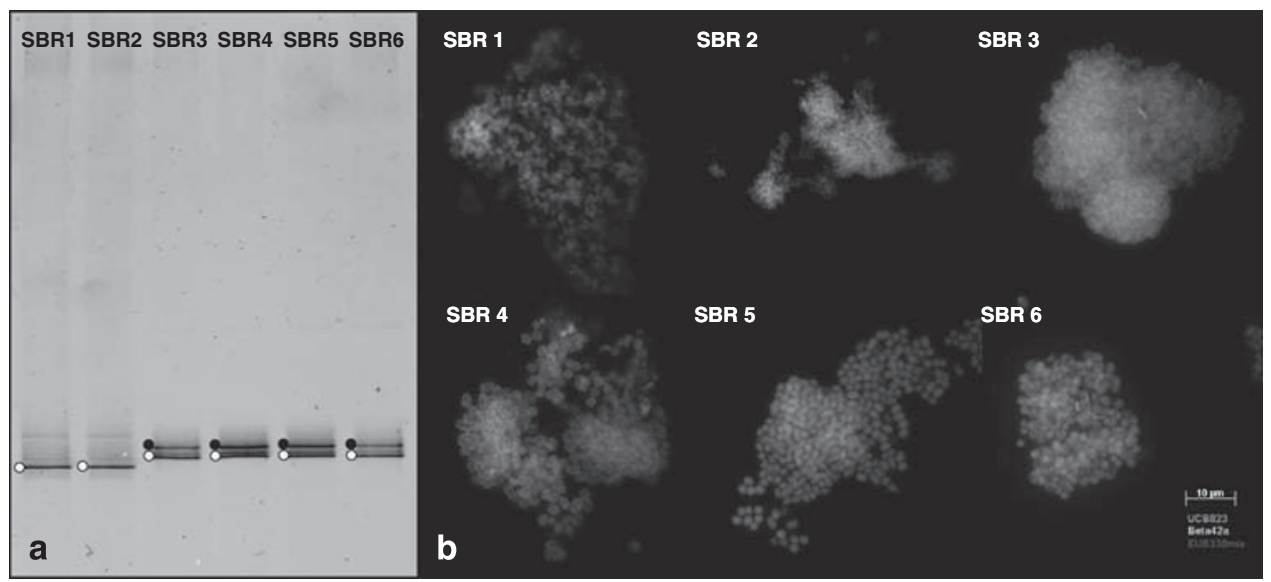

Figure 1 DGGE gel and FISH microscopic photographs. (a) DGGE gel of bacterial 16S rRNA gene PCR products amplified from the mixed microbial communities of all SBRs. Lane numbers refer to SBR numbers. The bands labeled by white and black dots were excised and re-amplified for microbial identification. The sequences from the bands labeled with white dots were used for microbial species identification. From SBR 3 to 6, the sequences from the bands labeled with black dots were found identical to white dots-labeled bands. (b) FISH image of different enrichments stained with Cy5-labled probe for bacteria (EUB338mix, blue), fluorescein-labeled probe for Betaproteobacteria (Beta42a, green) and Cy3-labeled specific probe for P. acidivorans (UCB823, red). The light blue color indicates that both EUB338mix and Beta42a hybridized. The purple color indicates both EUB338mix and UCB823 hybridized.

enrichment efficiency of dominant bacterial species during the enrichment stage cannot be ruled out. Because $P$. acidivorans is a novel bacterial species with superior PHB-producing capacity, and a specific primer (or probe) for Zoogloea is not available, this work focused on analyzing the development of $P$. acidivorans during the enrichment stage.

According to the results of microbial composition analysis, $P$. acidivorans could only be enriched in the $30{ }^{\circ} \mathrm{C}$ SBRs. Therefore, the enrichment efficiency of $P$. acidivorans was only investigated in reactors operated at $30^{\circ} \mathrm{C}$ (SBR 6 was not investigated). The relative abundance of $P$. acidivorans in total bacterial species in these three SBRs (SBR 3, SBR 4 and SBR 5) was continuously analyzed for 7 days since the first day of bioaugmentation. We found that $P$. acidivorans can be enriched to more than $80 \%$ of the total microbial community within six SRTs in all analyzed SBRs (Figures 2a and c). The curves of enriching $P$. acidivorans can be described by a logistic equation (Equation (2)).

$$
X_{t}=\frac{X_{0} \cdot e^{\alpha t}}{1+X_{0} \cdot e^{\alpha t}}
$$

In this equation, $X_{0}$ and $X_{\mathrm{t}}$ indicate the relative abundance of $P$. acidivorans at time zero and time point $t$, respectively. The symbol $\alpha$ effectively indicates the $P$. acidivorans enrichment efficiency. No significant variation in $\alpha$ could be identified as a function of the cycle length (Figures 2a and c), indicating that the cycle length had no direct impact on the $P$. acidivorans enrichment efficiency.

\section{Short-term temperature change experiment}

To explain the dominance of Zoogloea in the SBRs at $20^{\circ} \mathrm{C}$ and the dominance of $P$. acidivorans in the
SBRs at $30^{\circ} \mathrm{C}$, the effect of temperature change on two different enrichments was examined. Biomass from SBR 2 cultivated at $20^{\circ} \mathrm{C}$ and dominated by Zoogloea and SBR 5 cultivated at $30^{\circ} \mathrm{C}$ and dominated by $P$. acidivorans were chosen for the short-term temperature change experiment. In this experiment, we changed the temperature of SBR 2 to $30^{\circ} \mathrm{C}$ and the temperature of SBR 5 to $20^{\circ} \mathrm{C}$ for one cycle under non-steady state. These two experiments are presented as SBR 2* and SBR 5* in Table 1.

Both enrichments showed a higher biomassspecific reaction rate at $30^{\circ} \mathrm{C}$. Increasing the temperature by $10^{\circ} \mathrm{C}$, substrate uptake rate and $k$-value of $P$. acidivorans were increased by a factor of 2.11 and 4, respectively. Within the same temperature change, the substrate uptake rate and $k$-value of Zoogloea were increased by a factor of 1.11 and 1.95, respectively. Comparing the performance of two enrichments at the same temperature, $P$. acidivorans was characterized by a much higher substrate uptake rate during the feast phase and a lower PHB degradation rate during the famine phase as compared with Zoogloea.

To verify the temperature sensitivity of the two types of biomass, we studied the respiration capacity of the biomass from SBR 2 and SBR 5 in a wider temperature range $\left(15\right.$ to $\left.30^{\circ} \mathrm{C}\right)$. Within the temperature range tested, the oxygen uptake rate of both enrichments showed a clear trend, which can be accurately described by the simplified Arrhenius equation (Equation (1)). The oxygen uptake rate temperature coefficient $(\theta)$ for $P$. acidivorans and Zoogloea was 1.061 and 1.047, respectively ( $R^{2}$ values of $0.97-0.99$, Figure 3$)$. According to the values of $\theta$, the oxygen uptake rate of $P$. acidivorans and Zoogloea increased by 1.8 and 1.6 times, respectively, by increasing the temperature from 20 to $30{ }^{\circ} \mathrm{C}$. 

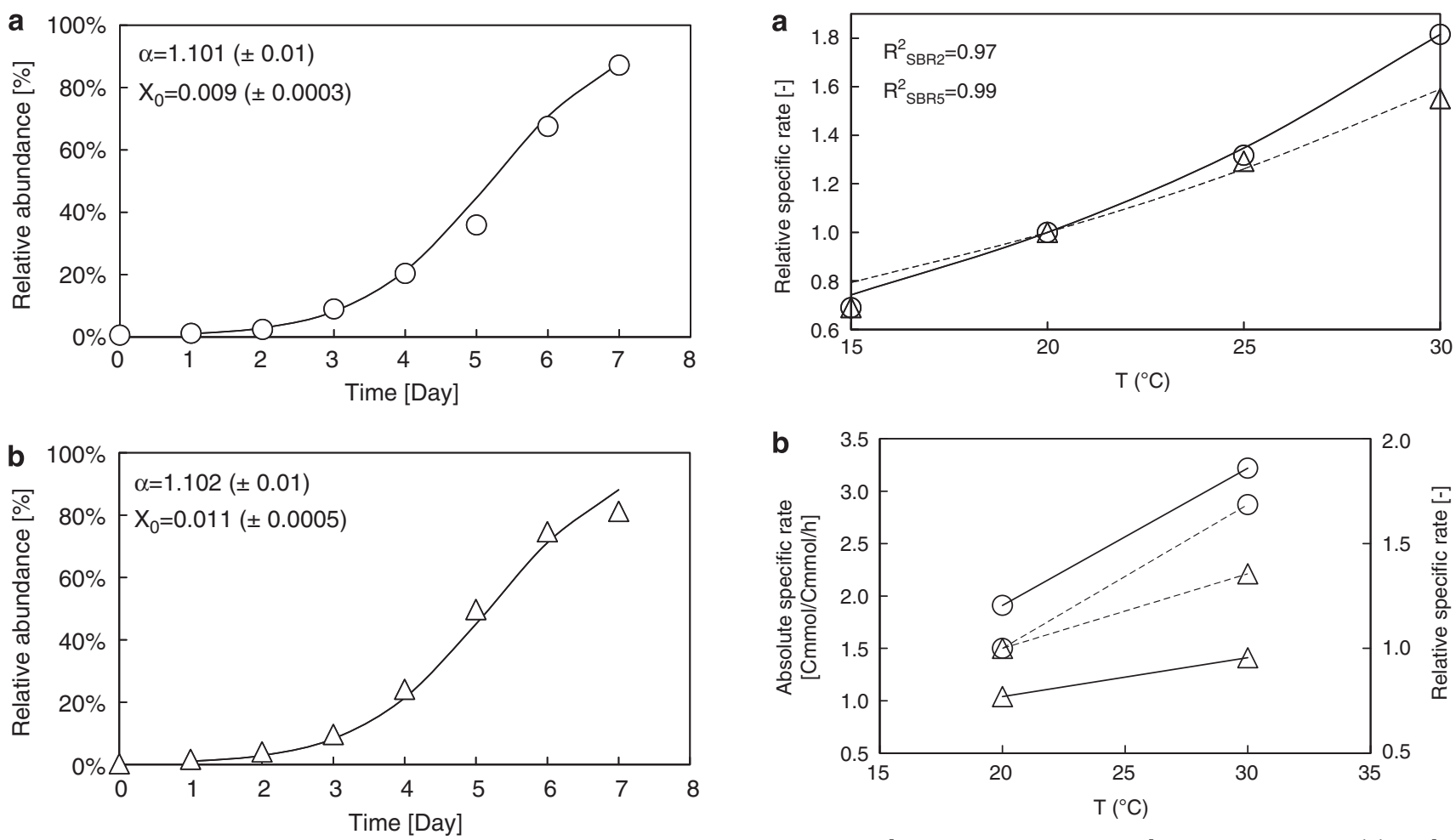

Figure 3 Short-term temperature change experiment. (a) Arrhenius efficiency obtained from the biological oxygen monitor experiments. Symbol (experimental data), lines (modeled data). Circle and solid line indicate the data from $P$. acidovorans enrichment. Triangle and dashed line represent the data from Zoogloea enrichment. (b) Absolute and relative comparison of substrate uptake rate of $P$. acidivorans (circle) and Zoogloea (triangle) when increasing temperature by $10^{\circ} \mathrm{C}$. Solid lines indicate the absolute increase of substrate uptake rate and dashed lines represent the relative increase of substrate uptake rate.

length of the SBRs. By increasing the cycle length from 1 to $18 \mathrm{~h}$, the PHB content at the end of the feast phase was increased from $5.6 \%$ to $71.3 \%$ (Table 1 ).

Figure 2 qPCR analysis of the relative abundance of $P$. acidivorans during enrichments in feast-famine SBRs. Symbols indicate the relative abundance of $P$. acidivorans over total bacteria from the experimental data. The solid lines indicate the model data by logistic equation. $\alpha$ indicates the $P$. acidivorans enrichment efficiency. (a) SBR $1 \mathrm{~h}$ cycle length, $30^{\circ} \mathrm{C}$, (b) SBR $4 \mathrm{~h}$ cycle length, $30^{\circ} \mathrm{C}$, (c) SBR $12 \mathrm{~h}$ cycle length, $30^{\circ} \mathrm{C}$.

Combining the results of the substrate uptake rate, the $k$-value and the oxygen uptake rate, $P$. acidivorans was found to be more temperature sensitive. The largest difference between two enrichments was observed from $k$-value, associated with PHA degradation rate.

Influence of the cycle length on the SBR performance Independent of the microbial composition of the enrichments and the temperature, cycle length showed a clear influence on the performance of SBRs. It was observed that the PHB content at the end of the feast phase was associated with the cycle

\section{Fed-batch experiments}

Fed-batch experiments were performed under nitrogen-limiting conditions to investigate the maximum PHB storage capacity of different enrichments. Biomass from SBR 2 and SBR 5 was used for fedbatch experiments at their respective enriched temperature. The PHB accumulation over time is presented in Figure 4. Both enrichments showed superior PHB-producing capacity: the culture from SBR 2 reached about $72 \mathrm{wt} \%$ PHB within $11 \mathrm{~h}$. The culture enriched in SBR 5 showed a very high PHB storage capacity and reached more than $80 \mathrm{wt} \%$ PHB after just over $4 \mathrm{~h}$.

\section{Discussion}

Influence of temperature on microbial competition The main objective of this study is to understand the microbial competition between $P$. acidivorans and 


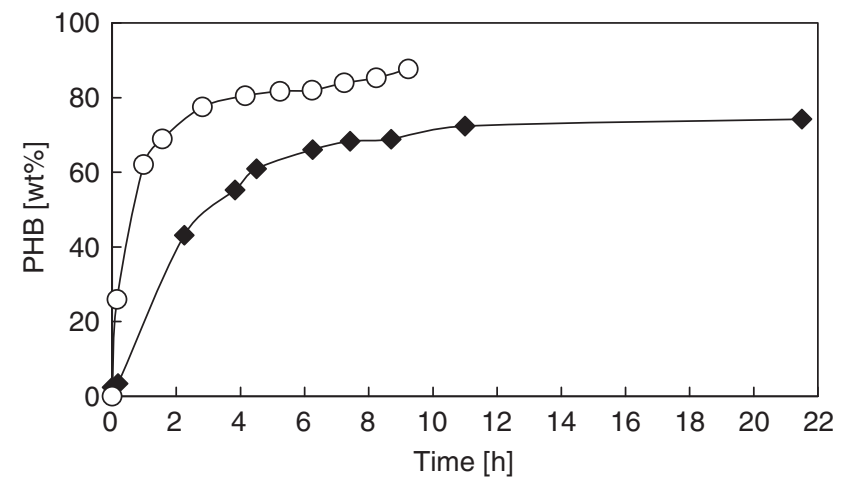

Figure 4 PHB content as function of time in PHB accumulation experiments. Filled diamond indicates the fed-batch experiment of SBR2 $\left(12 \mathrm{~h}, 20^{\circ} \mathrm{C}\right.$, Zoogloea $)$ operated at $20^{\circ} \mathrm{C}$. Non-filled circle indicates the fed-batch experiment of SBR5 $\left(12 \mathrm{~h}, 30^{\circ} \mathrm{C}, P\right.$. acidovorans) operated at $30^{\circ} \mathrm{C}$.

other bacterial species under feast-famine conditions. The experiments clearly demonstrated that an operational temperature of $30^{\circ} \mathrm{C}$ was the crucial factor for enrichment of $P$. acidivorans. Compared with the main competitor found in these experiments, Zoogloea, P. acidivorans has a strong competitive advantage in terms of the biomass-specific substrate uptake rate. This is particularly the case at the higher temperature investigated $\left(30^{\circ} \mathrm{C}\right.$, see Table 1).

On the basis of the substrate uptake rate alone, it can be expected that $P$. acidivorans could outcompete Zoogloea as well at the lower temperature investigated $\left(20^{\circ} \mathrm{C}\right)$. The observation is not the case, and Zoogloea dominated the systems operated at $20^{\circ} \mathrm{C}$, which is likely due to the temperature effect on PHB degradation of $P$. acidivorans. As indicated by the $k$-value and the PHB content at the end of SBR cycle (Table 1), P. acidivorans had very low PHB degradation rate and incomplete PHB degradation during the famine phase. Incomplete PHB degradation of $P$. acidivorans limits the overall growth rate in the system at $20^{\circ} \mathrm{C}$. The incomplete conversion of PHB to biomass provides a strong competitive disadvantage to $P$. acidivorans compared with Zoogloea. According to the $k$-value of $P$. acidivorans at $20^{\circ} \mathrm{C}$, it was estimated that $P$. acidivorans needs a minimal SRT of 6 days to accomplish complete PHB to biomass conversion at this temperature.

The effect of temperature on the PHA-producing mixed cultures has been documented before. Johnson et al., 2010 studied the temperature influence on the kinetics in a very similar system as in our study. However, the microbial competition was not studied.

Krishna and van Loosdrecht, 1999 also evaluated the effect of temperatures ranging from 15 to $35^{\circ} \mathrm{C}$ in PHA-producing mixed cultures. Unlike our observation, they found Zoogloea-like bacteria as dominant species in their reactor operated at $30^{\circ} \mathrm{C}$. This different finding might be the result of a different feeding strategy applied by these authors: the length

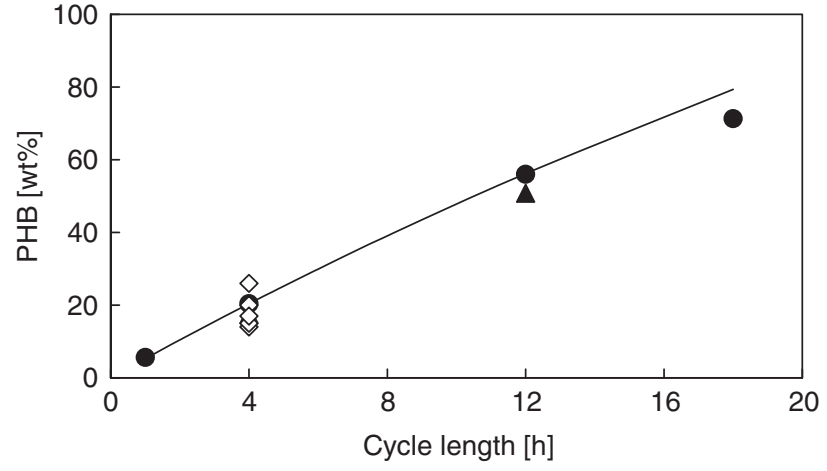

Figure 5 PHB content at the end of feast phase in SBRs with feast-famine process. line (modeled data by Equation (9) in Appendix B); full filled circle (experimental data from $30^{\circ} \mathrm{C}$ SBRs in this study); full filled triangle (experimental data from $20^{\circ} \mathrm{C}$ SBRs in this study); Non-filled diamond (experimental data from $4 \mathrm{~h}$ cycle length SBR operated at different temperature in Johnson et al., 2010).

of the feast phase was controlled by the substrate supply rate resulting in substrate limiting conditions during the feast phase. In that case, the microorganisms are selected based on the affinity for the substrate (Ks), rather than by the maximum substrate uptake rate. From a cell morphology point of view, Zoogloea may have a higher substrate affinity compared with $P$. acidivorans, because $P$. acidivorans cells were significantly larger than Zoogloea $(1.8 \mu \mathrm{m}$ vs $0.6 \mu \mathrm{m}$ diameter). Larger cells have a lower surface to volume ratio, suggesting that $P$. acidivorans may have a lower substrate affinity (Matin and Veldkamp, 1978). Additionally, the use of a different inoculum might be another explanation for the absence of $P$. acidivorans in their system.

Number of cycles per SRT as a new selection criterion for optimized PHA production

The PHB content at the end of the feast phase was associated to the cycle length, independent of the temperature and the microbial community structure in the SBR, and can be predicted by Equation (9) (Appendix B). In this equation, the molecular weights of active biomass $M w_{X}$ and PHB $M w_{P H B}$ and the yield coefficient of active biomass on PHB $Y_{X, P H B}$ in the famine phase can be assumed constant. The PHB content is therefore only determined by the ratio of SRT over cycle length, which is defined as the number of cycles per SRT. The lower cycle number per SRT is, the higher the PHB content that needs to be established during the feast phase.

The measured cellular PHB contents at the end of the feast phase in all SBRs operated in this study were very similar to the estimated value as calculated using the method shown in Appendix B. The experimental data from other SBRs operated in a similar manner (Johnson et al., 2010) were additionally used to validate Equation (9). As can be seen from Figure 5, the experimental data on the PHB 
content at the end of feast phase can be described by Equation (9) very well. In SBR 6 (18 h), bacteria have to accumulate more than $70 \mathrm{wt} \%$ PHB during the feast phase. Under these conditions, bacteria incapable of establishing such a high cellular PHBcontent are likely to be outcompeted.

Together with a constant SRT, a prolonged cycle length results in a higher PHB content at the end of the feast phase. There are two factors contributing to this: On one hand, the amount of substrate fed per cycle is increased and biomass concentration at the beginning of the cycle in the reactor decreased by prolonging the cycle length (or minimizing the cycle number per SRT). Consequently, the substrate to biomass ratio increases, leading to more $\mathrm{PHB}$ synthesis. On the other hand, the famine phase is prolonged. Cells need more PHB to survive during the prolonged famine phase. Both aspects intensify the selection of microorganisms with a high substrate uptake rate and high storage capacity. The number of cycles per SRT is therefore proposed as a useful selection pressure for the effective enrichment of a microbial community, with the capacity to establish a high PHB content at a high rate.

The influence of cycle length on PHA production has been sparsely studied. Most groups use a relatively large number of cycles per SRT in their experiments, which leads to a low amount of PHB accumulated during the feast phase. Only Dionisi et al., 2007 conducted a similar study as reported here. They investigated the influence of cycle length (from 1 to $8 \mathrm{~h}$ ) on the kinetic properties of the SBR enrichment cultures and the microbial community with a fixed SRT at day 1 and an organic loading rate at $20 \mathrm{gCOD} \mathrm{l}^{-1}$ per day, which is 10 times higher than our organic loading rate. The carbon source was a mixture of acetate, propionate and lactate. The temperature and $\mathrm{pH}$ were maintained at $25^{\circ} \mathrm{C}$ and pH 7.5 in their study. In contrast to our results, they found a strong influence of the cycle length on microbial composition. The higher organic loading rate might explain the different effect of cycle length on microbial composition. Because the amount of substrate fed per cycle was correlated with the cycle length, the substrate concentration, in their reactors operated with long cycle, was significantly higher. Consequently, bacteria may be inhibited or selected by the substrate concentration tolerance in their study.

The overall objective of this research is the production of PHA from agricultural waste. Several groups have been investigating this process using prefermented wastewater as substrate (Bengtsson et al., 2008; Albuquerque et al., 2010). The substrate composition in these studies was more complex than the substrate we used in our study. Whether $P$. acidivorans will be enriched on these substrates is still unknown. However, for process development purposes, the eventual microbial community obtained is unimportant as long as the functional stability (that is, PHB production) is ensured. The number of cycles per SRT can effectively select microorganisms based on their PHA-producing capacity, independent of substrate composition, temperature or $\mathrm{pH}$. The PHA-producing capacity is ensured by implementing this principle of selective pressure; either $P$. acidivorans or other types of superior PHA-producing bacterium can be enriched.

\section{Acknowledgements}

We thank Gert van der Steen for the analytical work. The investigation was supported by the Netherlands Organisation for Scientific Research (NWO) in the NWO-ACTS research programme B_BASIC and by the Foundation for Technical Sciences (STW).

\section{References}

Albuquerque MGE, Torres CAV, Reis MAM. (2010). Polyhydroxyalkanoate (PHA) production by a mixed microbial culture using sugar molasses: effect of the influent substrate concentration on culture selection. Water Res 44: 3419-3433.

Amann RI, Binder BJ, Olson RJ, Chisholm SW, Devereux R, Stahl DA. (1990). Combination of 16s ribosomal-RNAtargeted oligonucleotide probes with flow cytometry for analyzing mixed microbial populations. Appl Environ Microbiol 56: 1919-1925.

Bengtsson S, Werker A, Welander T. (2008). Production of polyhydroxyalkanoates by glycogen accumulating organisms treating a paper mill wastewater. Water Sci Technol 58: 323-330.

Beun JJ, Dircks K, van Loosdrecht MCM, Heijnen JJ. (2002). Poly-beta-hydroxybutyrate metabolism in dynamically fed mixed microbial cultures. Water Res 36: $1167-1180$.

Braunegg G, Lefebvre G, Genser KF. (1998). Polyhydroxyalkanoates, biopolyesters from renewable resources: physiological and engineering aspects. J Biotechnol 65: 127-161.

Brdjanovic D, van Loosdrecht MCM, Hooijmans CM, Alaerts GJ, Heijnen JJ. (1997). Temperature effects on physiology of biological phosphorus removal. J Environ Eng-Asce 123: 144-153.

Daims H, Bruhl A, Amann R, Schleifer KH, Wagner M. (1999). The domain-specific probe EUB338 is insufficient for the detection of all bacteria: development and evaluation of a more comprehensive probe set. Syst Appl Microbiol 22: 434-444.

Dias JML, Lemos PC, Serafim LS, Oliveira C, Eiroa M, Albuquerque MGE et al. (2006). Recent advances in polyhydroxyalkanoate production by mixed aerobic cultures: from the substrate to the final product. Macromol Biosci 6: 885-906.

Dionisi D, Majone M, Vallini G, Di Gregorio S, Beccari M. (2007). Effect of the length of the cycle on biodegradable polymer production and microbial community selection in a sequencing batch reactor. Biotechnol Progr 23: 1064-1073.

Johnson K, Jiang Y, Kleerebezem R, Muyzer G, van Loosdrecht MCM. (2009a). Enrichment of a mixed bacterial culture with a high polyhydroxyalkanoate storage capacity. Biomacromolecules 10: 670-676.

Johnson K, Kleerebezem R, van Loosdrecht MCM. (2009b). Model-based data evaluation of polyhydroxybutyrate 
producing mixed microbial cultures in aerobic sequencing batch and fed-batch reactors. Biotechnol Bioeng 104: 50-67.

Johnson K, van Geest J, Kleerebezem R, van Loosdrecht MCM. (2010). Short- and long-term temperature effects on aerobic polyhydroxybutyrate producing mixed cultures. Water Res 44: 1689-1700.

Keshavarz T, Roy I. (2010). Polyhydroxyalkanoates: bioplastics with a green agenda. Curr Opin Microbiol 13: 321-326.

Kleerebezem R, van Loosdrecht MCM. (2007). Mixed culture biotechnology for bioenergy production. Curr Opin Biotech 18: 207-212.

Kowalchuk GA, Stephen JR, DeBoer W, Prosser JI, Embley TM, Woldendorp JW. (1997). Analysis of ammonia-oxidizing bacteria of the beta subdivision of the class proteobacteria in coastal sand dunes by denaturing gradient gel electrophoresis and sequencing of PCR-amplified 16S ribosomal DNA fragments. Appl Environ Microbiol 63: 1489-1497.

Krishna C, van Loosdrecht MCM. (1999). Effect of temperature on storage polymers and settleability of activated sludge. Water Res 33: 2374-2382.

Lemoigne M. (1926). Produit de déshydratation et de polymérisation de l'acide $\beta$-oxybutyrique. Bulletin de la Société de chimie biologique 8: 770-782.

Manz W, Amann R, Ludwig W, Wagner M, Schleifer KH. (1992). Phylogenetic oligodeoxynucleotide probes for the major subclasses of proteobacteria: problems and solutions. Syst Appl Microbiol 15: 593-600.
Matin A, Veldkamp H. (1978). Physiological basis of selective advantage of a Spirillum sp. in a carbonlimited environment. J Gen Microbiol 105: 187-197.

Mudliar SN, Vaidya AN, Kumar MS, Dahikar S, Chakrabarti T. (2008). Techno-economic evaluation of PHB production from activated sludge. Clean Technol Environ Policy 10: 255-262.

Reis MAM, Serafim LS, Lemos PC, Ramos AM, Aguiar FR, Van Loosdrecht MCM. (2003). Production of polyhydroxyalkanoates by mixed microbial cultures. Bioprocess Biosyst Eng 25: 377-385.

Rossello-Mora RA, Wagner M, Amann R, Schleifer KH. (1995). The abundance of Zoogloea ramigera in sewage treatment plants. Appl Environ Microb 61: 702-707.

Schäfer H, Muyzer G. (2001). Denaturing gradient gel electrophoresis in marine microbial ecology. Method Microbiol 30: 425-468.

Slater SC, Voige WH, Dennis DE. (1988). Cloning and expression in Escherichia coli of the Alcaligenes eutrophus H16 poly-beta-hydroxybutyrate biosynthetic-pathway. J Bacteriol 170: 4431-4436.

Steinbüchel A. (1991). Polyhydroxyalkanoic acids. In: Byrom D (ed). Biomaterials novel materials from biological sources. Stockton: New York, 124-213.

van Loosdrecht MCM. (2000). Method for the production of polyhydroxyalkanoate. European Patent Office, WO0052189.

Vishniac W, Santer M. (1957). Thiobacilli. Bacteriol Rev 21: 195-213. 


\section{Appendix A}

Sequencing-batch reactors (stable operation and non-stable operation) cycle behaviors at different temperature and cycle length. (a) SBR 1, 4-h cycle length, $20^{\circ} \mathrm{C}$, stable, Zoogloea. (b) SBR 2, 12-h cycle
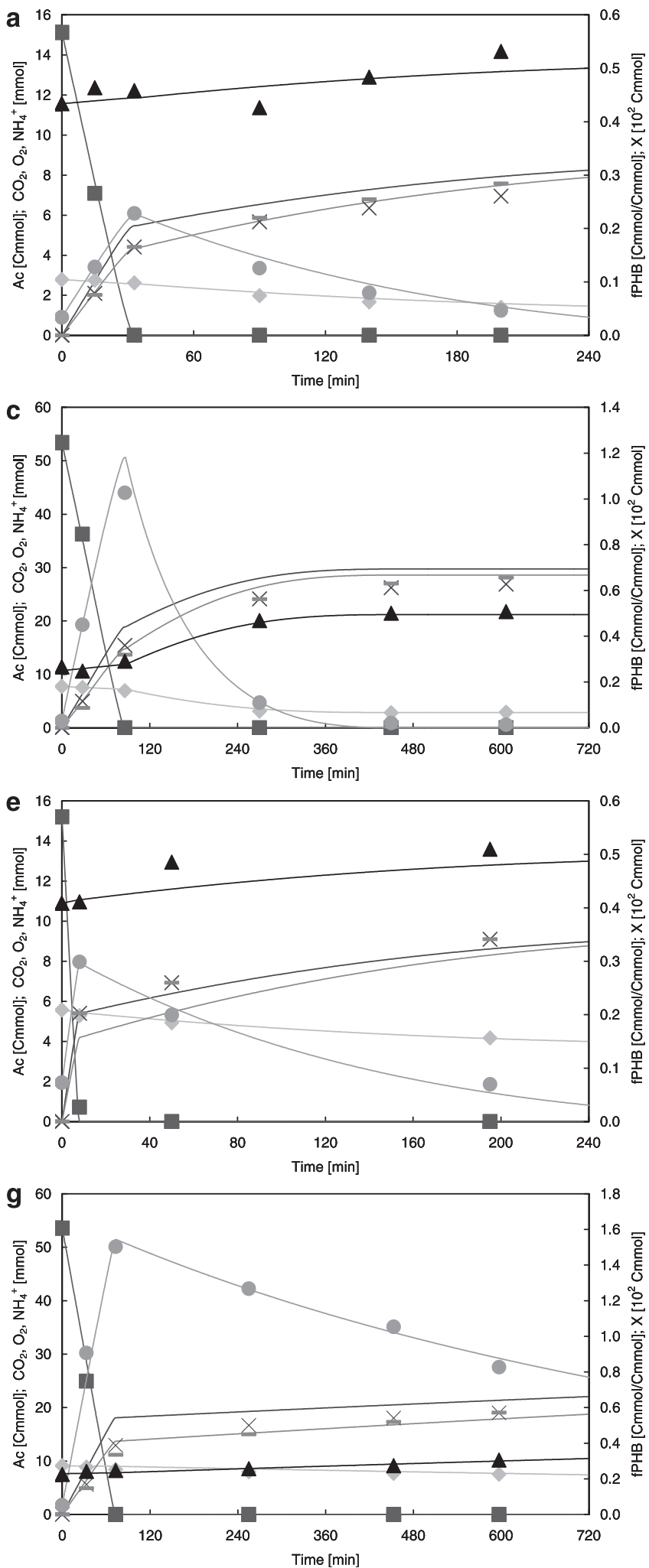

length, $20^{\circ} \mathrm{C}$, stable, Zoogloea. (c) SBR 2*, 12-h cycle length, $30^{\circ} \mathrm{C}$, non-stable, Zoogloea. (d) SBR 3, 1-h cycle length, $30^{\circ} \mathrm{C}$, stable, $P$. acidivorans. (e) SBR 4, 4-h cycle length, $30^{\circ} \mathrm{C}$, stable, P. acidivorans. (f) SBR 5, 12-h cycle length, $30^{\circ} \mathrm{C}$, stable, P. acidivorans. (g) SBR $5 *$, 12 -h cycle length, $20^{\circ} \mathrm{C}$,
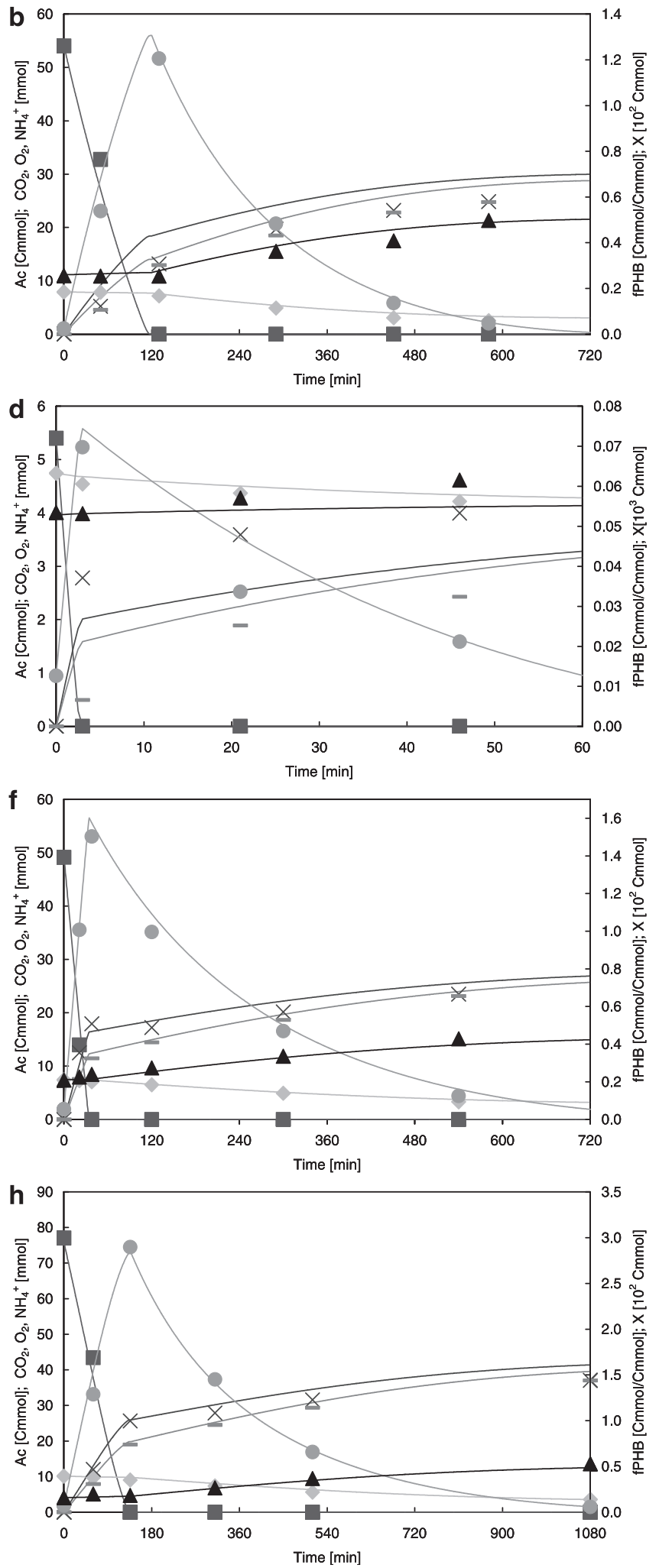
non-stable, $P$. acidivorans. (h) SBR 6, 18-h cycle length, $30^{\circ} \mathrm{C}$, stable, $P$. acidivorans. Solid lines indicate modeled values corresponding to the respective symbols indicating measurements.

\section{Appendix B}

Model construction

To analyze the impact of the cycle length on the process, the following simplifications are proposed: (1) there is no growth in the feast phase, (2) maintenance can be neglected for cycle analysis and (3) the polyhydroxybutyrate (PHB) accumulated in the feast phase is completely consumed in the famine phase. In other words, all new generated active biomass during the famine is assumed to be derived from stored PHB during the feast phase. Using this approach the cellular PHB content at the end of the feast phase can be predicted:

The active biomass concentration at the end of the cycle can be calculated by the initial active biomass concentration at the beginning of the cycle, SRT and cycle length (equation (3), Johnson et al., 2009b).

$$
\begin{gathered}
C_{\mathrm{x}}^{\text {end }}=C_{\mathrm{x}}^{\text {initial }} \cdot \frac{\mathrm{SRT}}{\mathrm{SRT}-\mathrm{CL}} \\
\quad \text { expressed in }\left(\mathrm{Cmol} \mathrm{l}^{-1}\right)
\end{gathered}
$$

Thereby, the total new generated active biomass concentration is the difference between $\mathrm{C}_{\mathrm{x}}^{\text {end }}$ and $\mathrm{C}_{\mathrm{x}}^{\text {initial }}$ (equation (4))

$$
\begin{aligned}
& C_{\mathrm{x}}^{\text {new }}=C_{\mathrm{x}}^{\text {end }}-C_{\mathrm{x}}^{\text {initial }} \\
& \quad \text { expressed in }\left(\mathrm{Cmol} \mathrm{l}^{-1}\right)
\end{aligned}
$$

Because all new generated active biomass is produced from stored PHB during the feast phase, the PHB concentration needed can be calculated according to the stoichiometry yield of biomass over PHB ( $Y_{\mathrm{x}, \mathrm{PHB}}$, equation (5)).

$$
\begin{gathered}
C_{\mathrm{PHB}}=\frac{C_{\mathrm{x}}^{\mathrm{end}}-C_{\mathrm{x}}^{\text {initial }}}{Y_{\mathrm{X}, \mathrm{PHB}}} \\
\text { expressed in }\left(\mathrm{Cmol} \mathrm{l}^{-1}\right)
\end{gathered}
$$

Acetate; (○) PHB; ( $\mathbf{\Delta}$ ) active biomass; $(\diamond)$ ammonia; $(\times)$ cumulative carbon dioxide evolution; $(\square)$ cumulative oxygen uptake. Note: The scales of the axes are not all consistent.

The unit can be converted to weight concentration $\left(\mathrm{g}^{-1}\right)$ by multiplying the molecular weight of PHB per carbon mole $\left(M w_{\mathrm{PHB}}\right.$, equation (6))

$$
\begin{aligned}
& \mathrm{C}_{\mathrm{PHB}, \mathrm{g} \mathrm{l}^{-1}}=\frac{\left[\mathrm{C}_{\mathrm{x}}^{\mathrm{end}}-\mathrm{C}_{\mathrm{x}}^{\text {initial }}\right] \cdot M w_{\mathrm{PHB}}}{Y_{\mathrm{X}, \mathrm{PHB}}} \\
& \text { expressed in }\left(\mathrm{g}^{-1}\right)
\end{aligned}
$$

In the steady-state assumed, no growth occurs during the feast phase. The concentration of active biomass at the end of the feast phase should be the same as the initial active biomass concentration. By multiplying the molecular weight of active biomass per carbon mole, the unit can be converted to gram per liter (equation (7))

$$
C_{\mathrm{x}, \mathrm{g} 1^{-1}}^{\text {initial }}=C_{\mathrm{x}}^{\text {initial }} \times M w_{\mathrm{x}} \quad \text { expressed in }\left(\mathrm{gl}^{-1}\right)
$$

The PHB content is the weight of PHB over total solid suspension, which is composed by PHB and active biomass. The PHB content at the end of the feast phase can be expressed by equation (8).

$$
\frac{\mathrm{PHB}^{\text {feast }} w t \%=C_{\mathrm{PHB}, \mathrm{gl}^{-1}}}{C_{\mathrm{PHB}, \mathrm{g} l^{-1}}+C_{\mathrm{x}, \mathrm{g} l^{-1}}^{\text {initial }}}
$$

By combining equation $(6,7)$ into equation $(8)$, the cellular PHB content at the end of the feast phase can be estimated equation (9):

$$
\mathrm{PHB}^{\text {feast }} w t \%=\frac{1}{1+\left(\frac{\mathrm{SRT}}{\mathrm{CL}}-1\right) \cdot \frac{M w_{\mathrm{X}} \cdot Y_{\mathrm{X}, \mathrm{PHB}}}{M w_{\mathrm{PHB}}}}
$$

